\title{
Lista actualizada de aves dañinas en Costa Rica (2012)
}

\author{
Javier Monge \\ Centro de Investigaciones en Protección de Cultivos (CIPROC), Escuela de Agronomía, Universidad de Costa Rica; javier.monge@ucr.ac.cr
}

Recibido 20-IV-2012 Corregido 7-IX-2012 Aceptado 29-X-2012

\begin{abstract}
Updated checklist of harmful birds in Costa Rica (2012). This is an updated list of birds that are considered harmful in Costa Rica, mainly for agricultural production. This update considers recent information, both the status of harmful species as changes in the taxonomy of some species. The list consists of 63 species belonging to 20 families, mainly Icteridae, Psittacidae, Emberizidae, Columbidae and Cardinalidae. Sorghum, rice and maize are more frequently affected.There is a need for diagnostic studies of the current situation of bird pests, with special reference to the impact assessment as a criterion for implementing management plans.
\end{abstract}

\section{KEY WORDS}

Vertebrate pest, pest birds, damage, crop production, Icteridae, Psittacidae.

\section{RESUMEN}

En este documento se actualiza a 2012 la lista de aves que se consideran dañinas en Costa Rica, principalmente para la producción agrícola. Esta actualización considera información reciente, tanto de la condición de especie dañina como de cambios en la taxonomía de algunas especies. La lista está constituida por 63 especies, pertenecientes a 20 familias, siendo las más importantes las familias Icteridae, Psittacidae, Emberizidae, Columbidae y Cardinalidae. Los cultivos más frecuentemente afectados son los granos (sorgo, arroz y maíz). Urgen estudios sobre impacto como criterio para decidir si se justifica implementar planes de manejo.

\section{PALABRAS CLAVE}

Vertebrados plaga, aves plaga, daños, producción agrícola, Icteridae, Psittacidae.
El conflicto entre las aves y el ser humano es quizás tan antiguo como la agricultura. Entre los daños que ocasionan las aves se encuentran el consumo, contaminación o destrucción de comestibles; pérdidas de productos no comestibles; transmisión de enfermedades; accidentes de aviación y averías en aviones; daños en construcciones, comodidad para el uso de sitios públicos o estética de fachadas de edificios o monumentos; y daños al ambiente (Monge, 2007).

En el caso de Costa Rica se cuenta con un listado de aves plaga (Hilje y Monge, 1988) pero es algo anacrónico, por lo que amerita una actualización, incorporando nueva información no solo acerca de su importancia económica, sino también sobre su taxonomía. Para los propósitos de este documento, se considera como plaga a aquella "especie de mamífero, ave, reptil, anfibio o pez que afecta directa o indirectamente a la especie humana, ya sea porque provoque daños en las áreas de producción, consuma o contamine alimentos almacenados, cause daños en la infraestructura, transmita enfermedades o provoque la muerte a personas o animales domésticos" (Monge, 2007).

Una de las limitantes de este tema es la carencia de datos de pérdidas provocados por aves, ya que, muchas de las referencias se enfocan a la descripción del daño. Aún cuando en algunos países latinoamericanos se han hecho estudios para evaluar los métodos de control, existen muy pocos estudios que evalúan los daños provocados por las aves (Sedano, 2003). Esta situación limita que muchas de las especies enlistadas, se les pueda considerar como plaga en forma objetiva, situación válida para otros países latinoamericanos (Bucher, 1984).

En cuanto a la información disponible referente a la magnitud de los daños provocados por las aves, se tiene la 
percepción de que los productores tienden a magnificar los daños producidos por las aves, lo cual puede estar influenciado por lo conspicuo de algunas especies de aves, como es el caso de loras y pericos (Bucher, 1998)

El propósito de este documento es presentar información actualizada acerca de las especies de aves que se consideran dañinas en Costa Rica, incluyéndose aquellas que afectan la producción agrícola, con algunas referencias al impacto a la producción pecuaria y acuícola. Puesto que muchas de ellas aparecen en otros países de América y el Caribe, dicha información podría ser útil para los productores y técnicos que laboran en dichos sectores productivos fuera de Costa Rica.

\section{METODOLOGÍA}

Para la presente recopilación se recurrió a varios tipos de fuentes bibliográficas. En primer lugar, a la verificación de la lista de Hilje y Monge (1988), complementada con observaciones del autor y comunicaciones personales, debidamente verificadas, de productores y profesionales. Como referencias de otros ámbitos geográficos, pero pertinentes, aunque también muy generales, se contó con las publicaciones de De Grazio y Besser (1970), De Grazio (1978), y Elias y Valencia (1984), además de diferentes referencias latinoamericanas sobre especies o cultivos en particular. Asimismo, en la Guía de aves de Costa Rica de Stiles y Skutch (2003), para algunas especies se usa el calificativo de especie plaga o dañina, o se mencionan algunos cultivos que forman parte de su dieta. Los nombres científicos corresponden a los incluidos en la lista oficial de aves de Costa Rica actualizada al 2011 (Asociación Ornitológica de Costa Rica, 2012; Unión de Ornitólogos de Costa Rica, 2012), mientras que los nombres comunes son los utilizados en Costa Rica o los sugeridos por Stiles y Skutch (2003). El uso de nombres comunes sirve de referencia para los no especialistas en aves, aunque tiene el inconveniente que dichos nombres no siempre son de uso generalizado o bien diferentes especies pueden ser conocidas por el mismo nombre común, como el caso del "pius".

El hecho de que una especie aparezca aquí enlistada no significa que sea plaga en la actualidad, sino que potencialmente podría alcanzar dicha condición, en función de las características de los sistemas de producción, de las condiciones ambientales o de aspectos intrínsecos de las especies involucradas.

\section{RESULTADOS}

La lista de aves consideradas dañinas presentes en Costa Rica, la constituyen 62 especies, pertenecientes a 20 familias, lo cual sugiere que la variedad de daños e impactos es amplia. Es importante tener presente la diferencia entre especie que alcanza la condición de plaga y especie dañina. En el primer caso se refiere a una condición temporal o permanente en que una población de una determinada especie ocasiona un impacto económico importante, criterio que puede variar entre productores, dependiendo de las expectativas de rentabilidad de su actividad productiva. Sin embargo, en términos generales, para que ocurra un impacto significativo, es necesaria la participación de una cantidad importante de aves, de tal manera que en conjunto el impacto sea cuantioso. Por lo contrario, en casos de un impacto bajo, provocado por unas pocas aves, no debería de considerarse el calificativo de plaga, sino quizás el de especie dañina.

A continuación se presenta la lista de especies de aves presentes en Costa Rica, de las cuales se ha reportado que ocasionan daños a la producción agrícola, en general. En los casos en que se dispone de información de la evaluación del impacto, se incluye, aunque no necesariamente corresponde a estudios realizados en Costa Rica. A su vez, aún reconociendo el valor de la estimación del daño, debe tenerse presente que éste se presenta en diferente magnitud en función de las características del sistema de producción evaluado, así como el estado de las poblaciones de la especie implicada en el sitio en donde se hace la valoración del impacto.

\section{Anatidae (Patos, gansos, cisnes)}

Dendrocygna viduata (piche careto). Su daño consiste en la extracción de plántulas para comer semillas de arroz y el pisoteo de las plántulas en busca de alimentos. Su condición de especie dañina ha sido reconocida también en Nicaragua, Venezuela, Surinam, Colombia y Uruguay. El arroz representó una parte importante en la dieta de esta especie, de aproximadamente una tercera parte, en un estudio realizado en arrozales en Calabozo, Venezuela (Bruzual \& Bruzual, 1983).

Dendrocygna autumnalis (piche o pijije). Esta especie, al igual que otros patos silvestres, con los que ocasionalmente forma grandes bandadas, afecta el arroz, al extraer las plántulas para consumir la semilla o por el pisoteo en busca de alimento, entre los que también se encuentran los insectos acuáticos. En Venezuela se reporta que junto con Dendrocyga viduata y Dendrocygna bicolor causa daños considerables al consumir el grano de arroz pregerminado (Poleo \& Mendoza, 2000), lo cual contrasta con lo 
indicado por Bourne y Osborne (1978) quienes estimaron un consumo entre 0,2 y $2 \%$, por lo que consideran que el impacto no es perjudicial. Bruzual y Bruzual (1983) estimaron que un $80 \%$ de la dieta de esta especie en arrozales de Calabozo, Venezuela, está constituida precisamente por arroz. Una situación similar fue encontrada en Sinaloa, México, en donde el arroz representó una parte muy importante de su dieta (Kramer \& Euliss, 1986). Para Costa Rica fue reportado como una plaga seria para el cultivo de arroz, principalmente cuando el arroz está emergiendo (De Grazio, 1984).

Dendrocygna bicolor (piche). Su daño se debe a la extracción de plántulas para comer semillas de arroz y por el pisoteo de las plántulas en busca de alimento. En Venezuela, se estimó que esta especie junto con $D$. viduata causa pérdidas de 1,2 a 1,6\% en época lluviosa y de 6,2 a $9,0 \%$ en época seca, para un promedio de 1,8 a $2 \%$ para todo el año (Casler et al., 1981). Bruzual y Bruzual (1983) estimaron que el arroz representó el $68 \%$ de la dieta de esta especie según un estudio realizado en arrozales en Calabozo, Venezuela.

Cairina moschata (pato real). Se alimenta principalmente en áreas abiertas, incluyendo pantanos y áreas agrícolas con maíz y arroz (Besser, 1968) y ocasionalmente consume tubérculos. En Tamaulipas, México, donde las siembras de granos son comunes, el maíz constituye la dieta completa de mayo a agosto (Bolen, 1991).

Anas discors (zarceta). Su daño se debe a la extracción de plántulas, consumo de semillas y pisoteo de arroz. Este daño ha sido reconocido en Costa Rica y Venezuela.

\section{Phalacrocoracidae (cormoranes)}

Phalacrocorax brasilianus (cormorán). El cormorán se alimenta principalmente de peces, los cuales los encuentra en forma concentrada y abundante en los estanques, por lo puede llega a ocasionar pérdidas importantes para la acuacultura. Tiene predilección por peces relativamente pequeños o medianos, por lo que los estanques con peces jóvenes son más afectados. Pueden afectar el crecimiento de los peces debido al estrés que les provocan (Contreras et al., 2003). Los peces grandes pueden ser afectados, aún cuando no siempre los logra atrapar, puede ocasionarles heridas importantes. También son considerados perjudiciales para la pesca deportiva y comercial (Bucher, 1984).

\section{Ardeidae (garzas)}

Ardea alba (garceta grande). Se alimenta de peces, siendo por la depredación de éstos en los estanques que se le considera especie dañina.
Egretta caerulea (garceta azul). Se considera dañina por la depredación de peces de estanques.

Bubulcus ibis (garza bueyera). Provoca pisoteo en los cultivos de arroz, durante sus recorridos en busca de insectos acuáticos para su alimentación.

\section{Cathartidae (zopilotes)}

Coragyps atratus (zopilote o zoncho). Su dieta es muy variada, incluyendo componentes vegetales y animales, $y$ principalmente carroña. Afecta el banano y la palma africana, consumiendo parte de la producción en los sitios de acopio de fruta; esto ocurre también en Honduras y Colombia (Elias \& Valencia, 1982). Además, atacan terneros recién nacidos, aunque en forma esporádica.

Cathartes aura (zopilote de cabeza roja o zoncho). Afecta la palma africana, al consumir parte de la producción de fruta. Este daño ha sido reconocido en Colombia y Honduras (Sánchez \& Ortiz, 1998).

\section{Pandionidae (águila pescadora)}

Pandion haliaetus (águila pescadora). El águila pescadora, como su nombre lo indica, es un depredador de peces, aunque también se alimenta de otros vertebrados pequeños. Para su alimentación requiere de aguas tranquilas con peces que naden cerca de la superficie, condición que le ofrecen los estanques, por lo que se convierte en una especie dañina para la acuacultura.

\section{Accipitridae (águilas, gavilanes)}

Buteo platypterus (gavilán pollero). Su dieta incluye reptiles, insectos grandes, mamíferos pequeños y ocasionalmente aves pequeñas. Es precisamente por la depredación de aves de corral que se le considera dañina. En Perú, haciéndose referencia al género Buteo, incluyendo 10 especies, se indica que ataca aves de corral, ocasionando daños de diferente magnitud (Aguilar et al., 1977).

\section{Rallidae (gallareta)}

Porphyrio martinico (gallina de agua). Esta especie tiene una dieta variada que incluye frutos de los lotos y otras plantas acuáticas, arroz y otros granos, insectos acuáticos y otros invertebrados y peces pequeños. Los daños se presentan en el cultivo de arroz, principalmente por el pisoteo que ocasiona en estos campos. Otro daño mecánico lo provocan al utilizar el follaje del cultivo para la construcción de nidos (Poleo \& Mendoza, 2000). Agüero et al. (2005) se refieren a un daño mecánico de esta especie y 
Gallinula galeata en tan solo 0,076\% evaluado en una parcela de arroz de 2,2 has en Venezuela.

Gallinula galeata (gallareta frenti-roja). Esta especie ha sido considerada dañina en Venezuela y Colombia por los daños que ocasiona al cultivo de maíz, además al arroz por la construcción de nidos o plataformas con el follaje de este cultivo. En Colombia, el daño mecánico al arroz se estimó entre 3,4 y $12,7 \%$, mientras que por consumo no se reportan daños (Sedano, 2003).

\section{Jacanidae (jacanas)}

Jacana jacana (jacana). La jacana daña cultivos de arroz en Centroamérica y Suramérica (Elias \& Valencia, 1984). Los ambientes acuáticos, como los campos de arroz anegados, son ambientes comúnmente frecuentados por esta especie.

\section{Columbidae (palomas)}

Patagioenas flavirostris (paloma morada). Ha sido referida como una plaga local en Costa Rica, dado que consume plántulas de maíz y sorgo (Stiles \& Skutch, 2003).

Patagioenas fasciata (paloma collareja). Esta especie ha sido considerada dañina para el cultivo de arroz en varios países de Suramérica, tales como Colombia, Ecuador, Perú y Venezuela (Agüero et al., 2005). En Venezuela, también se reporta de daños en áreas de producción de semillas de arvejas y trigo (Aguilar, 2000). En Costa Rica, aún no ha sido reportado como plaga.

Zenaida asiatica (paloma de ala blanca). Se considera una especie dañina para el cultivo de trigo, en varios países de Latinoamérica, tales como Argentina, Nicaragua, México y Honduras (Elias y Valencia, 1984). También se considera dañina para el cultivo de sorgo en México, algunos países de Centroamérica y de Suramérica (Besser, 1968; De Grazio \& Besser, 1970; Poleo \& Mendoza, 2000). En Costa Rica, aún no ha sido reportada como especie dañina, ni tampoco se hace referencia que habiten en campos agrícolas, pero si en sabanas de la provincia de Guanacaste. Puede formar grandes bandadas de individuos migratorios de Norteamérica que invernan en Guanacaste entre noviembre y mayo.

Columbina passerina (tortolita, palomita). Al igual que las otras palomas referidas, los daños son provocados en sorgo y arroz, lo cual ha sido documentado en Colombia y Venezuela (Elias \& Valencia, 1984). De igual manera, aún cuando en Costa Rica no ha sido referida como una especie dañina, si se indica que habita en tierras cultivadas.
Columbina minuta (tortolita). Esta especie provoca daños a los cultivos de sorgo y arroz, situación que ha sido reportada en Colombia y Venezuela (Elias \& Valencia, 1984), pero aún no en Costa Rica.

Columbina talpacoti (tortolita o palomita colorada). Los daños de esta paloma son provocados al sorgo y arroz, daños que han sido reconocidos en Colombia, Venezuela (Elias \& Valencia, 1984) y El Salvador. En Costa Rica, aún cuando no ha sido documentado este tipo de daños, se hace referencia que habita en sitios abiertos incluyendo áreas agrícolas.

\section{Psittacidae (loras, pericos)}

Aratinga finschi (cotorra / perico frenti-rojo). Este perico ha sido considerado en Costa Rica como una plaga para los cultivos de maíz y sorgo (De Grazio, 1984).

Aratinga canicularis (catano, zapoyol, perico frenti-anaranjado). Este perico daña cultivos de sorgo y maíz, situación que ha sido reportada para Costa Rica, Nicaragua, Venezuela y Colombia (Besser, 1968; De Grazio \& Besser, 1970; Elias \& Valencia, 1984).

Aratinga pertinax (perico carasucio). Esta especie recién descrita para Costa Rica, ha sido reportada como dañina en cultivos de maíz, ajonjolí y sorgo en Venezuela (Albornoz \& Fernández-Badillo, 1994), reportándose pérdidas de hasta un 40,71\% en maíz (García, 1986). Si hasta hace poco ha sido descubierta en Costa Rica sugiere que sus poblaciones no son numerosas, por lo que el riesgo de que se convierta en plaga, por lo pronto es bajo.

Brotogeris jugularis (catano, perico barba-anaranjada). Este perico se alimenta de diversos frutos silvestres, pero también se le reconoce como plaga para el cultivo de maíz. En Puntarenas, Costa Rica, se estimaron pérdidas que en algunos casos llegaron al 100\%, según estimaciones hechas por los productores (Guido, 1992)

Pionus menstruus (chucuyo). Este perico es dañino por consumir granos de maíz y frutos de banano y pejibaye. Este último daño también es reportado para Colombia (Pava et al, 1983).

Pionus senilis (chucuyo, loro coroniblanco). Este perico es considerado una plaga seria en cultivos de maíz, pejibaye, sorgo y frutas en maduración, situación reconocida tanto en Costa Rica como en Honduras y Colombia (De Grazio, 1984; Elias \& Valencia, 1984; Pava et al., 1983). También se considera dañina para el cultivo de arroz. En México se estimó un nivel de daño de 3,3 \% en maíz (RomeroBanderas et al., 2006).

Amazona albifrons (lora, loro frenti-blanco). El loro frente-blanco es una especie reconocida como dañina para el 
cultivo de maíz en Costa Rica, aunque también daña otros cultivos, entre los que se encuentra el mango.

Amazona autumnalis (lora, loro frenti-rojo). El loro frenti-rojo afecta cultivos de granos como el sorgo (Elias $\&$ Valencia, 1984), cultivo en que se ha sido documentada la presencia de bandadas de hasta 200 individuos, en Puntarenas, Costa Rica (De Grazio \& Besser, 1970). También dañan otros cultivos como el mango, naranja y aguacate. La producción de girasol también es dañada por esta especie. Los daños estimados en naranja oscilaron entre 0,91 y $5,41 \%$, en Guanacaste, Costa Rica (Chediak, 1999).

\section{Alcedinidae (martines pescadores)}

Chloroceryle amazona (martín pescador). Las especies conocidas con ese nombre común consumen peces (Poulin et al., 1994), por lo que afectan la producción acuícola. La situación no ha alcanzado hasta ahora dimensiones importantes, aunque ha sido reportada que afecta la producción de peces en Perú (Rebaza et al., 2002) y Colombia.

\section{Picidae (carpinteros)}

Melanerpes formicivorus (carpintero). Esta especie es considerada dañina por el consumo de maíz. Aún cuando se considera que los pájaros carpinteros afectan la madera, ya sea de árboles o en postes, existen referencias que indican que los árboles que utilizan son viejos, deteriorados, y hasta muertos, por lo que el hecho de que los picoteen en busca de invertebrados o para construir sus nidos, no necesariamente ha de representar un problema importante.

Melanerpes chrysauchen (carpintero nuquidorado). Este carpintero se alimenta de invertebrados y varios tipos de fruta, entre las que se encuentra el banano, aunque aún no se ha reportado como una especie dañina importante.

Dryocopus lineatus (carpintero lineado). En México se ha reportado que daña el maíz y su daño se ha estimado en 0,5\% (Romero-Banderas et al., 2006).

\section{Thamnophilidae (Batará, hormigueros)}

Thamnophilus doliatus (cacareona, batará listado). Esta especie ha sido reportada como dañina por el consumo de semillas de cardamomo, aun cuando su dieta se considera constituida principalmente por insectos.

\section{Tyrannidae (Mosqueros americanos)}

Pitangus sulphuratus (cristofué, pecho amarillo). Esta especie tiene una dieta variada incluyendo frutos y componentes animales, tales como lagartijas pequeñas, culebras, ranas, ratones, lombrices, arañas, y también peces. En Perú se considera dañino a la producción de peces (Rebaza et al., 2002), y en Uruguay es una de las especies que más daña la producción de uva (Rodríguez et al., 2004).

\section{Corvidae (Urracas, cuervos)}

Psilorhinus morio (piapia). Su dieta incluye frutos silvestres y cultivados. Se convierte en especie dañina al consumir granos de maíz y arveja, así como frutos de aguacate y zapallo y al depredar huevos de gallina. El daño en el cultivo de maíz estimado en México fue de tan solo 0,2\% (Romero-Banderas et al., 2006).

\section{Thraupidae (Tangara, viuda)}

Thraupis episcopus (viuda). Esta especie ha sido considerada dañina por el consumo de pulpa de manzanas.

Saltator coerulescens (sensontle o sinconte). Daña cultivos de sorgo en El Salvador, mientras que en Costa Rica solo se hace referencia que habita, entre otros ambientes, en jardines y cultivos sombreados, pero sin indicarse daños en algún cultivo en particular (Stiles \& Skutch, 2003).

\section{Emberizidae (Gorriones del nuevo mundo, picogruesos)}

Volatinia jacarina (pius). El pius daña cultivos sorgo y arroz, situación que ha sido reportada para El Salvador y Venezuela (Agüero et al., 2005). Para Costa Rica no se ha reportado como plaga de algún cultivo agrícola.

Sporophila torqueola (semillero, espiguero collarejo). Esta especie es dañina por consumir granos de arroz y sorgo, siendo referida en Costa Rica como una especie que veces es una plaga en los campos de estos cultivos (De Grazio, 1984; Stiles \& Skutch, 2003).

Sporophila minuta (espiguero pigmeo). Esta especie ha sido considera dañina dado que consume granos de arroz y sorgo, situación que ha sido reportada en México, Centroamérica y Suramérica (Elias \& Valencia, 1984).

Sicalis luteola (chirigüe sabanero). Daña cultivos de sorgo en Uruguay y Argentina (Bucher, 1984); sin embargo, en Costa Rica no se hace referencia a su presencia en campos agrícolas, ni a daños a cultivos en particular (Stiles \& Skutch, 2003).

Emberizoides herbicola (sabanero coludo). Esta especie ha dañado cultivos de sorgo en Colombia y Venezuela (Agüero et al., 2005). En Costa Rica, aún no ha sido 
reportado como especie dañina, sino que se considera que frecuenta sabanas con gramíneas en donde busca insectos y semillas (Stiles \& Skutch, 2003).

Chondestes grammacus (gorrión arlequín). Esta especie ha sido recién encontrada en Costa Rica (Stiles \& Skutch, 2003), la cual ha sido considerada como dañina en México, al ser observada en cientos, comiendo sorgo cosechado (De Grazio \& Besser, 1970).

Zonotrichia capensis (comemaíz). Esta especie causa daños en trigo en Colombia (De Grazio \& Besser, 1970) y en Uruguay daña la producción de uva (Rodríguez et al., 2004). Aún cuando en Costa Rica no se ha referido como una plaga importante para algún cultivo, si se reconoce que frecuente, entre otros ambientes, los campos agrícolas.

\section{Cardinalidae (saltador, picogrueso)}

Piranga flava (tangara veranera). Esta especie afecta los cítricos, al picotear sus frutos.

Pheucticus ludovicianus (calandria). La calandria daña cultivos de sorgo en El Salvador, aunque en Costa Rica no hay referencias de posibles daños que ocasiona a la producción agrícola (Stiles \& Skutch, 2003).

Passerina caerulea (picogrueso azul). Daña cultivos de sorgo en El Salvador. En Costa Rica se indica que frecuenta áreas agrícolas, entre otros ambientes, pero no se hace referencia a daños en algún cultivo en particular (Stiles \& Skutch, 2003).

Passerina cyanea (indris o azulillo norteño). Esta especie daña cultivos de sorgo en El Salvador. En Costa Rica sólo se hace referencia a que frecuentan cultivos enmalezados y campos agrícolas entre otros ambientes, pero no se indica que ocasionen daños a algún cultivo en particular (Stiles \& Skutch, 2003).

Passerina ciris (siete colores). Daña cultivos de sorgo en El Salvador, pero no se dispone de referencias de daños que ocasiona a cultivos en Costa Rica.

Spiza americana (pius, sabanero). El pius o sabanero daña arroz y sorgo en maduración en Latinoamérica (De Grazio \& Besser, 1970; 1974), incluyendo Centroamérica, Venezuela, Colombia y Perú. Su impacto es muy importante dado que es altamente gregario y puede llegar a forma grandes bandadas. Tiene predilección por áreas despejadas con zacate Panicum maximun, así como por arrozales. En Venezuela, se informa que se alimenta básicamente de semillas de arroz, sorgo y pastos silvestres (Poleo \& Fuentes, 2005). En el estado Portuguesa, Venezuela, se estima un daño de $2,4 \%$ en sorgo y de $1,3 \%$ en arroz (Agüero et al., 2005).

\section{Icteridae (oropéndolas, caciques, pájaros negros)}

Dolichonyx oryzivorus (tordo arrocero). Esta especie se alimenta principalmente de semillas de hierbas y zacate, aunque también incluye al arroz dentro de su dieta, por lo cual se le considera especie dañina tanto en Costa Rica, como en otros países de Centroamérica y Suramérica (Bucher, 1984; Elias \& Valencia, 1984; Agüero et al., 2005). En Venezuela, se reportaron pérdidas del 30\% (De Grazio \& Besser, 1970). También se considera que es una especie dañina para el maíz.

Agelaius phoeniceus (sargento). El sargento es una especie reconocida como dañina para varios cultivos de granos como arroz, sorgo y maíz, lo cual ha ocurrido en Costa Rica, Honduras y México (De Grazio \& Besser, 1970; Elias $\&$ Valencia, 1984). En México, se estimó pérdidas en esos cultivos hasta en un 40\% (Del Villar-González et al., 2006), aunque en el cultivo de arroz en Morelos, México, ésta junto con otras especies ocasionaron un daño de 3,68\% (Del Villar \& Acosta, 2002).

Sturnella militaris (tordo pechirrojo). Esta especie se ha reportado como dañina para los cultivos de arroz y sorgo en Colombia y Uruguay. Para Costa Rica no se ha hecho referencia a su condición dañina, aunque se le encuentra en arrozales.

Xanthocephalus xanthocephalus (tordo cabecidorado). Esta especie daña cultivos de sorgo, arroz, trigo, garbanzo y frijol, situación que ha sido reportada en México (De Grazio \& Besser, 1970; Elias \& Valencia, 1984). En Costa Rica, no se hecho referencia a su condición dañina para algún cultivo, aunque se le puede encontrar en campos de arroz anegados. A menudo se encuentra en asociación con bandadas de sargentos (A. phoeniceus), los cuales si son reconocidos como plaga en el arroz.

Dives dives (tordo cantor). Esta especie puede atacar mazorcas de maíz en maduración y además ha sido reportada como dañina al cultivo de sorgo (Chiri, 1988).

Quiscalus mexicanus (zanate). El zanate tiene una dieta muy variada, aprovechando diferentes recursos que encuentra en ambientes agrícolas o urbanos. En los campos agrícolas sus daños son diversos, afectando diferentes estadíos de los cultivos; al consumir semillas o extraer las plántulas para consumir la semilla, en maíz, arroz, sorgo y melón, razón por la cual es considera una plaga importante (Besser, 1968; De Grazio, 1984; Arnold, 1991). También puede afectarlos cuando se han producido el grano y se está pronto a cosechar, situación que ocurre en Costa Rica, y ha sido documentado para México y Honduras. Otros reportes hacen referencia a la extracción de semillas de maní y Jatropha curcas, especie cultivada para la producción de biocombustibles, daño que se generaliza a otros cultivos. 
Molothrus aeneus (pius). Esta especie ha sido reportada como dañina para el cultivo del sorgo en El Salvador, así como tomate, sorgo, maíz y arroz en México (Del VillarGonzález et al., 2006). En Morelos, México, se estimó que junto con otras especies ocasionó un 3,68\% de pérdidas en arroz (Del Villar \& Acosta, 2002). En Costa Rica, solo se ha hecho referencia a que frecuenta áreas abiertas, especialmente campos agrícolas, por lo que podría esperarse que ocasione algún tipo de daños en éstos.

Icterus galbula (cacique veranero). Esta especie tiene una dieta variada que incluye néctar de varias especies silvestres, aunque también se alimenta de bananos y otras frutas, sin que necesariamente llegue a representar un problema importante. Sin embargo, se considera que a veces llega a ser una dañina en cultivos de tomate y cítricos.

Psarocolius montezuma (oropéndola de Montezuma). Esta especie se alimenta de bastantes frutos y semillas ariladas, incluyendo bananos maduros de cultivo, por lo que se le puede considerar como una especie dañina. También se ha considerado dañina para el cultivo de pejibaye.

\section{Passeridae (gorriones del Viejo Mundo)}

Passer domesticus (gorrión común). Esta especie ha sido considerada plaga para los cultivos de sorgo, maíz, arroz y trigo en México, mientras que en Uruguay daña la producción de uva (Rodríguez et al., 2004). Sin embargo, en Costa Rica no ha sido reportada como plaga, quizás por encontrarse principalmente en pueblos y ciudades, lo cual puede obedecer a su relativamente reciente invasión en el año 1974, aproximadamente (Stiles \& Skutch, 2003).

\section{DISCUSIÓN}

La cantidad de especies de aves presentes en Costa Rica consideradas plaga ya sea dentro del país, como en otros países representan aproximadamente el $7 \%$ de la avifauna de Costa Rica. La pertenencia de estas especies a 20 familias sugieren una diversidad importante, lo cual se refleja en una variedad de tipos de producción afectadas por las aves.

La familia más representada es Icteridae, de la cual se han reportado nueve especies dañinas, las que afectan cultivos muy variados, entre los que sobresalen los granos como arroz, maíz y sorgo, aunque también algunas especies dañan cítricos, melón y tomate. En esta familia se encuentra el zanate (Quiscalus mexicanus), importante plaga de diferentes cultivos, así como en zonas urbanas. Dada la formación de grandes parvadas por parte de varias especies de esta familia y su amplia distribución en la mayoría de las áreas agrícolas en México, se le considera como los principales vertebrados plaga (Urbina, 1993, citado por del Villar-González et al., 2006).

La segunda familia más representada es Psittacidae, la cual está constituida por las loras y pericos, con ocho especies reportadas como dañinas, principalmente de granos como maíz y sorgo. Estas especies tiene la particularidad de ser muy vistosas que vuelan en bandadas bulliciosas (Stiles \& Skutch, 2003), desde sus sitios de descanso hasta los sitios de alimentación en forma muy conspicua, lo cual puede influir que sean fácilmente detectadas ocasionando daños, y por ende referidas con frecuencia como especies dañinas. Esta situación también puede influir en la percepción del impacto que ocasionan en la agricultura (Bucher, 1998).

La siguiente familia en cuanto a representación es Emberizidae, la cual agrupa a aquellas aves referidas como gorriones del Nuevo Mundo, picogrueso y especies afines, tales como espigueros y sabaneros. Estas especies afectan principalmente granos como el maíz y particularmente el sorgo. Stiles y Skutch (2003) indican que los representantes nativos de esta familia tienden a alimentarse en sitios bajos, a menudo de manera oculta sobre el suelo debajo de matorrales, recogiendo semillas, insectos y frutas. Estas condiciones las ofrecen diversos cultivos, siendo el sorgo el cultivo más referido como afectado por más especies de esta familia, seguido por el arroz. Aunque esas referencias para Costa Rica son escasas, lo cual se puede relacionar con la poca producción del sorgo, esta información es útil en caso de interés por desarrollar este cultivo, principalmente para la elaboración de alimento para animales.

Las siguientes dos familias más representadas, en ambos casos por seis especies, son Cardinalidae y Columbidae. Las especies reportadas como dañinas de la familia Cardinalidae, conocidos como picogrueso, dañan los cultivos de sorgo. Dentro de esta familia se encuentra el pius o sabanero (Spiza americana), especie capaz de constituir grandes bandadas, las cuales se alojan en áreas de cultivo de arroz, y al aprovechar este recurso alimenticio la convierten en una importante plaga. En el caso de la familia Columbidae (palomas) también atacan principalmente cultivos de granos como el arroz y el sorgo.

En el caso de la familia Anatidae, que agrupa a patos, gansos y cisnes, la mayoría de las especies reportadas como dañinas, ocasionan daños en el cultivo de arroz, ya sea por consumir sus granos o semillas, así como por el pisoteo al cultivo en sus primeras etapas, cuando buscan insectos acuáticos en los campos anegados. Dichas especies forman bandadas de tamaño considerable, las cuales salen en grupos a alimentarse de noche (Stiles \& Skutch, 
2003). Su impacto se incrementa, dado el tamaño y peso de estas aves que ocasionan daños físicos importantes en los campos de cultivo.

El resto de las familias tiene una representación de apenas una a tres especies, lo cual no significa que su daño no sea importante, como puede ser el caso de los cormoranes (Phalacrocorax brasilianus) en la producción acuícola.

Con respecto a los cultivos, los granos son los que se reportan como más afectados por diferentes especies de aves. En el caso del sorgo, hay 29 especies que lo afectan, aunque no necesariamente en Costa Rica. El siguiente cultivo más afectado es el arroz, por al menos 24 especies, seguido del maíz, por 18 especies. Otros productos agrícolas afectados por aves son frutos, tales como banano, por cinco especies, y cítricos y pejibaye por tres especies (Cuadro 1).

En el caso de la acuacultura, se reportan daños ocasionados por seis especies de aves, siendo su impacto en algunos casos muy importante, dada la concentración de peces de un solo tamaño en estanques particulares, situación que los hace muy vulnerables, cuando coinciden con el tamaño de presas buscadas por diferentes especies de aves piscícolas.

Al analizar la información existente referente a las especies de aves dañinas en Costa Rica, es claro que existe algún nivel de conocimiento sobre la problemática generada por estas especies a los intereses del ser humano, aunque es relativamente escaso. Así, por ejemplo, en la mayoría de los casos se limita al reconocimiento de la especie dañina, sin que se disponga de información relacionada con la magnitud del daño, así como alguna caracterización de la problemática, en relación con la época en que ocurre, comportamiento de los individuos, dinámica poblacional de las especies, y menos aún acerca de resultados de prácticas de manejo.

De alguna manera, la sistematización de la información existente, su actualización y la utilización de aquella proveniente de otras latitudes con condiciones similares a las existentes en Costa Rica, permite disponer de información útil acerca del tema de las aves dañinas. Procede, en el mejor de los casos, utilizar dicha información, para identificar aquellas especies con mayor prioridad para ser estudiadas, ya sea por la importancia del sector productivo afectado, o por la magnitud reconocida, aunque no documentada, de los daños. Sin embargo, es conveniente realizar esfuerzos que permitan diagnosticar la problemática actual generada por diferentes especies de aves, dando énfasis a la evaluación de su impacto. Esta información daría fundamento para la toma de decisiones para implementar planes de manejo cuando la situación lo amerite.
CUADRO 1

Cantidad de especies por familia que atacan los cultivos más afectados

\begin{tabular}{|c|c|c|c|c|c|c|c|}
\hline Familia & 음 & $\frac{N}{\frac{N}{2}}$ & $\frac{N}{N}$ & 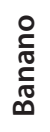 & 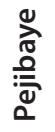 & $\underset{\mathscr{d}}{\mathscr{Q}}$ & $\begin{array}{l}\text { ò } \\
\text { ot }\end{array}$ \\
\hline Anatidae & & 5 & 1 & & & & \\
\hline Phalacrocoracidae & & & & & & 1 & \\
\hline Ardeidae & & 1 & & & & 2 & \\
\hline Cathartidae & & & & 1 & & & 1 \\
\hline Pandionidae & & & & & & 1 & \\
\hline Accipitridae & & & & & & & 1 \\
\hline Rallidae & & 2 & 1 & & & & \\
\hline Jacanidae & & 1 & & & & & \\
\hline Columbidae & 5 & 4 & 1 & & & & \\
\hline Psittacidae & 5 & & 7 & 1 & 2 & & \\
\hline Alcedinidae & & & & & & 1 & \\
\hline Picidae & & & 2 & 1 & & & \\
\hline Thamnophilidae & & & & & & & 1 \\
\hline Tyrannidae & & & & & & 1 & \\
\hline Corvidae & & & 1 & & & & \\
\hline Thraupidae & 1 & & & & & & 1 \\
\hline Emberizidae & 7 & 3 & & & & & \\
\hline Cardinalidae & 5 & 1 & & & & & \\
\hline Icteridae & 5 & 6 & 4 & 2 & 1 & & \\
\hline Passeridae & 1 & 1 & 1 & & & & \\
\hline TOTAL & 29 & 24 & 18 & 5 & 3 & 6 & 4 \\
\hline
\end{tabular}

\section{REFERENCIAS}

Agüero, D.A, Poleo, J. \& Duno, G. (2005). Especies de aves plaga. En: Plagas vertebradas. Fundación Nacional del Arroz: Venezuela.

Aguilar, H. (2000). La paloma torcasa o gargantilla Columba fasciata Say 1823 (Aves:Columbidae). Algunas notas sobre su historia natural en Venezuela. Revista de Ecología Latinoamericana $7(3), 29-44$

Aguilar, P.G., Beingolea, O.D., Brack, A.J. \& Ceballos, B. (1977). Vertebrados importantes en la agricultura peruana. Revista Peruana de Entomología 20(1),25-32.

Albornoz, M. \& Fernández, A. (1994). Psitácidos (Aves: Psittaciformes) plagas de cultivos en el valle de río Güey, estado Aragua, Venezuela. Revista de la Facultad de Agronomía 20,123-132. 
Arnold, K.A. (1991). Quiscalus mexicanus (zanate, Zanatles, sanate, Great-tarled Grackle). En: Janzen, D. (ed). Historia Natural de Costa Rica. Editorial de la Universidad de Costa Rica: San José, Costa Rica.

Asociación Ornitológica de Costa Rica. (2012). Lista oficial de aves de Costa Rica 2011. Recuperado de http://listaoficialavesdecostarica.files.wordpress.com/2012/03/lista-oficialaves-de-cr-2011-aocr.xls.

Besser, J.F. (1968). Impresions of bird damage problems in Mexico, Honduras, Nicaragua, Colombia and Venezuela. Trip Report, June, 1968. Unpublished report. Denver Wildlife Research Center: Denver, Colorado, EEUU.

Bolen, E.G. (1991). Cairina moschata (pato real, pato de alas blancas, moscovita, Muscovy Duck). En: Janzen, D. (ed). Historia Natural de Costa Rica. Editorial de la Universidad de Costa Rica: San José, Costa Rica.

Bourne, G.R. \& Osborne, D.R. (1978). Black-bellied whistling duck utilization of a rice culture habitat. Interciencia 3(3),152-159.

Bruzual, J., Bruzual, I. (1983). Feeding habits of whistling ducks in the Calabozo ricefields, Venezuela, during the reproductive period. Wildfowl 34,20-26.

Bucher, E.H. (1984). Las aves como plaga en la Argentina. Centro de Ecología Aplicada. Publicación №9. Facultad de Ciencias Exactas, Físicas y Naturales. Universidad de Córdoba: Cordoba, Argentina.

Bucher, E. (1998). Criterios básicos para el manejo integrado de aves plaga. En: Rodríguez, E.N. \& Zaccagnini, M.E. (Eds.) Manual de capacitación sobre manejo integrado de aves perjudiciales a la agricultura. FAO.

Casler, C.L., Rivero, A.R. \& Lira, J.R. (1981). Los patos Dendrocygna como causantes de daños en los cultivos de arroz de Venezuela (Aves, Anatidae). Memoria Sociedad de Ciencias Naturales La Salle 115,105-115.

Chediack, A. (1999). Diversidad y abundancia de aves en cultivos de mandarinas en Tucumán (Argentina) y comparación del daño causado por aves en cítricos dulces entre Argentina y Costa Rica. Tesis inédita de Maestría en Manejo de Vida Silvestre. Universidad Nacional: Heredia, Costa Rica.

Chiri, A.A. (1988). Los vertebrados como plaga de los cultivos en América latina. Manejo Integrado de Plagas (Costa Rica) 7,69-79.

Contreras, A.J., Tejeda, A.G. \& García, J.A. (2003). Las aves como plaga, controles y manejo. Ciencia UANL 1(1),93-98.

De Grazio, J.W. (1978). World bird damage problems. pp. 9-24. En: Marsh, R. E. (ed). Proceedings $8^{\text {th }}$ Vertebrate Pest Conference. California, EEUU.

De Grazio, J.W. \& Besser, J.F. (1970). Bird damage problems in Latin America. pp. 162-167. En: Dana, R. H. (ed). Proceedings $4^{\text {th }}$ Vertebrate Pest Conference. West Sacramento, California, EEUU.
De Grazio JW, Besser JF. (1974). Los pájaros silvestres se comen millones de dólares. Agricultura de las Américas 23(6), 10,15,29.

De Grazio, J.W. (1984). Vertebrate pest problems of Ecuador, The Galápagos Islands, and Costa Rica. Trip Report. Denver Wildlife Research Center: Denver, Colorado.

Del Villar, D. \& Acosta, N. (2002). Assessment of bird damage to early-ripening rice in Cuautla, Morelos State, México. $p$ 157-160 En: Timm, R.M., Schmidt, R.H. (eds). Proceedings 20th Vertebrate Pest Conference. Davis, California, USA.

Del Villar-González, D., Villanueva-Jiménez, J.A. \& Martínez-Becerril, C. (2006). Repelencia alimenticia de extractos de éter etílico de nim (Azadirachta indica) en arroz palay, para el control del tordo ojirrojo (Molothrus aeneus). Agrociencia 40(2), 197-203.

Elias, D.J. \& Valencia, D. (1982). Inusual feeding behavior by a population of black vulture. Wilson Bulletin 94(2),214.

Elias, D.J. \& Valencia, D. (1984). La agricultura latinoamericana y los vertebrados plaga. Interciencia 9(4),223-229.

García, J.L. (1986). Estimación del daño ocasionado por pericos (Aratinga pertinax venezuelae Zimmer y Phelps) en sabanas de maíz al sur del estado Guárico, Venezuela. Agronomía Tropical 36(1-3):37-42.

Guido, M.Y. (1992). Caracterización de daño de pericos y loras (Aves: Psittacidae) en el cultivo de maíz (Zea mays L.) y evaluación de métodos para su combate en Cóbano, Puntarenas, Costa Rica. Tesis inédita de Maestría en Manejo de Vida Silvestre. Universidad Nacional: Heredia, Costa Rica.

Hilje, L. \& Monge, J. (1988). Lista preliminar y consideraciones generales acerca de los animales vertebrados plaga en Costa Rica. Manejo Integrado de Plagas (Costa Rica) 10,39-52.

Kramer, G.W. \& Euliss, N.H. (1986). Winter foods of black-bellied whistling-duck in Northwestern Mexico. Journal Wildlife Management 50(3),413-416.

Monge, J. (2007). ¿Qué son plagas vertebradas? Revista Agronomía Costarricense 31(2), 111-121.

Pava, J., González, A., Castillo, E. \& Patiño, H. (1983). Aspectos de interés fitosanitario de la palma de chontaduro Bactris gasipaes H.B.K. en algunas regiones del Valle del Choco. Acta Agronómica 33(1), 25-35.

Poleo, Y. \& Mendoza, R. (2000). Aves que afectan el cultivo de arroz. FONAIAP DIVULGA 67, 21-24. Recuperado de: http:// sian.inia.gob.ve/repositorio/revistas_tec/Fonaip.Divulga/ fd67/texto/poleo.htm.

Poleo, C. \& Fuentes, L. (2005). Aves plaga de los cultivos de arroz y sorgo en Venezuela. Revista Digital del Centro nacional de Investigaciones Agropecuarias de Venezuela (CENIAP): Venezuela. Recuperado de http://www.ceniap.gov.ve/ ceniaphoy/articulos/n9/arti/poleo_c.htm.

Poulin, B., Lefebvre, G. \& McNeil, R. (1994). Diets of land birds from Northeastern Venezuela. The Condor 96.354-367. 
Rebaza, C., Villasana, E., Rebaza, M. \& Deza, S. (2002). Influencia de tres densidades de siembra en el crecimiento de Piaractus brachypomus "Paco" en segunda fase de alevinaje en estanques seminaturales. Folia amazónica 13 (1-2), 121-134.

Romero-Banderas, K., Naranjo, E.J., Morales, H.H. \& Nigh, R.B. (2006). Daños causados por vertebrados silvestres al cultivo de maíz en la Selva Lacandona, Chiapas, México. Interciencia 31(4),276-283.

Rodríguez, E.N., Tiscornia, G. \& Tobin, M.E. (2004). Bird depredation in Uruguayan vineyards. P. 136-139. En: Timm, R.M. \& Gorenzel, W.P. (eds). Proceedings $21^{\text {th }}$ Vertebrate Pest Conference. Davis, California, EEUU.
Sánchez, S. \& Ortiz, C.F. (1998). Plagas y polinizadores de la palma aceitera en Tabasco, México. ASD Oil Palm Papers 18,25-28.

Sedano, R.E. (2003). Los humedales y la ocupación de aves en los cultivos de arroz. FORO 25-28. Recuperado de: http://ciatlibrary.ciat.cgiar.org/Articulos_Ciat/aves_humedal.pdf

Stiles, F.G. \& Skutch, A.F. (2003). Guía de aves de Costa Rica. Tercera edición. Instituto Nacional de Biodiversidad: Heredia, Costa Rica.

Unión de Ornitólogos de Costa Rica. (2012). Lista oficial de aves de Costa Rica. Recuperado de http://www.uniondeornitologos.com/index.php?option=com_content\&view=art icle\&id=249\%3Alista-de-aves-de-costa-rica-18-de-febrero-012\&catid=13\%3Abiblioteca-virtual\&ltemid=82. 